\title{
PHYSICS-DRIVEN STRUCTURED COSPARSE MODELING FOR SOURCE LOCALIZATION
}

\author{
Sangnam Nam and Rémi Gribonval
}

INRIA Rennes - Bretagne Atlantique, France

\begin{abstract}
Cosparse modeling is a recent alternative to sparse modeling, where the notion of dictionary is replaced by that of an analysis operator. When a known analysis operator is well adapted to describe the signals of interest, the model and associated algorithms can be used to solve inverse problems. Here we show how to derive an operator to model certain classes of signals that satisfy physical laws, such as the heat equation or the wave equation. We illustrate the approach on an acoustic inverse problem with a toy model of wave propagation and discuss its potential extensions and the challenges it raises.
\end{abstract}

Index Terms - Sparsity; cosparsity; structured cosparsity; dictionary; analysis operator; pursuit algorithm.

\section{INTRODUCTION}

The concept of sparsity lies at the heart of various techniques in signal processing. One of the most popular ways to express this idea is the sparse synthesis model. Mathematically, there is a dictionary $\mathbf{D} \in \mathbf{R}^{d \times n}$ such that every signal of interest $\mathbf{x} \in \mathbf{R}^{d}$ is well approximated as $\mathbf{x} \approx \mathbf{D} \mathbf{z}$ where $\|\mathbf{z}\|_{0} \ll d$.

\subsection{The cosparse model}

There is an interesting 'dual' formulation of the idea of sparsity [1]. Recently [2], this model was explicitly formulated and some of its aspects were studied systematically. In this formulation, the representation of a signal $\mathbf{x}$ is directly computed by applying a linear operator $\Omega \in \mathbf{R}^{p \times d}$. The resulting coefficient vector $\mathbf{z}=\boldsymbol{\Omega} \mathbf{x}$ is called the analysis representation of $\mathbf{x}$ and is subsequently assumed to be (approximately) sparse. As pointed out in [2], the special-ness of the signal $\mathrm{x}$ comes from the location of the many zero entries of $\Omega \mathrm{x}$, rather than from that of the few non-zero entries of $\Omega \mathrm{x}$. This is due to the fact that a zero entry in $\Omega \mathrm{x}$ is associated to the constraint that $\mathrm{x}$ is orthogonal to the associated row of $\boldsymbol{\Omega}$. Thus, in the analysis model the signal is rather "carved out" using $\Omega$ than built of few blocks (the atoms of $\mathbf{D}$ ) as in the synthesis model. To highlight the distinction between models, the number of zeros $p-\|\boldsymbol{\Omega} \mathbf{x}\|_{0}$ of the analysis representation

The authors are supported by the European Community's FP7-FET program, SMALL project, grant agreement no. 225913, and by Agence Nationale de la Recherche (ANR), project ECHANGE (ANR-08-EMER-006).
$\Omega \mathrm{x}$ is defined to be the cosparsity of $\mathrm{x}$ (with respect to $\Omega$ ). Informally, we say that $\mathrm{x}$ is cosparse or that $\mathrm{x}$ satisfies the cosparse analysis model when its cosparsity is large.

\subsection{Inverse problems, cosparsity, and cosparse recovery}

In the context of linear inverse problems where one observes $\mathbf{y} \approx \mathbf{M x}$ with $\mathrm{x}$ cosparse (with respect to some $\boldsymbol{\Omega}$ ), a new greedy algorithm called the Greedy Analysis Pursuit (GAP) was introduced $[2,3]$.

\subsection{Choosing analysis operators}

One quickly realizes that a very important problem regarding the model needs to be addressed: How does one choose the analysis operator $\Omega$ for a given class of signals?

Existing work performed with analysis operators essentially focused on operators chosen using intuitions on the nature of the signals at hand $[4,5,6]$. In fact, the best-known analysis operator can be designed with this approach; for the signals consisting of piecewise constant cartoon images, the horizontal or vertical neighboring pixels tend to have the same values. This leads to the analysis operator that is made of all the vertical and horizontal one-step differences, closely linked to the TV-norm. The curvelet and shearlet transforms $[6,7]$ are also designed operators to capture curvy features even though they are not explicitly designed with the cosparse model in mind.

Another approach, that is being pursued in recent works [8, 9,10 ] consists in learning $\Omega$ from training examples $\mathbf{x}_{i}$.

\subsection{Contributions}

In this work, we follow a different track: we derive analysis operators from the knowledge that the signals satisfy known physical laws. We introduce the main idea in Section 2 in a general context and then explore it in more details in the context of the wave equation. By introducing a natural notion of "structured cosparsity", we show that structured cosparse recovery can be exploited for inverse problems related to source localizations and/or reconstruction from a limited number of measures. A modification of the GAP algorithm [2, 3], called GReedy Analysis Structured Pursuit (GRASP) is proposed and we illustrate its performance with some elementary experimental results in Section 4. 


\section{PHYSICS-DRIVEN ANALYSIS OPERATORS}

Many signals of interest are physical quantities that satisfy certain physical laws. For example, let us consider the distribution of temperature $u(\mathbf{r}, t)$ in some homogeneous medium at location $\mathbf{r}$ at time $t$. Suppose that there are a few heat sources located in this space. Then we know that $u$ satisfies the heat equation $\frac{\partial u}{\partial t}-\alpha \Delta u=f$, where $f(\mathbf{r}, t)=0$ except at the locations of the sources and $\alpha$ is a constant. Similarly if $u(\mathbf{r}, t)$ denotes the pressure field at location $\mathbf{r} \in \mathbb{R}^{3}$ at time $t$ in a room, it is known that $u$ satisfies the Helmholtz equation

$$
\Delta u-\frac{1}{c^{2}} \frac{\partial^{2} u}{\partial t^{2}}=f
$$

in the interior of the room. Here, $f(\mathbf{r}, t)=0$ for every $\mathbf{r}$ that is not an active source location and for $t$ corresponding to the time during which the corresponding source is not active.

\subsection{Derivation of Cosparse Model: Discretization}

In both settings, under appropriate discretization $\mathrm{x}$ of the unknown $u, \mathbf{z}$ of the right-hand side $f$, and $\boldsymbol{\Omega}$ of the partial differential operator $\mathbf{L}:=\Delta-\frac{1}{c^{2}} \frac{\partial^{2}}{\partial t^{2}}\left(\right.$ resp. $\left.\mathbf{L}:=\frac{\partial}{\partial t}-\alpha \Delta\right)$, the signal of interest $\mathbf{x}$ satisfies

$$
\Omega_{\mathbf{X}}=\mathbf{z}
$$

for some $\mathbf{z}$ that is close to zero with few exceptions. Therefore, a cosparsifying operator $\Omega$ naturally arises from the equations through discretization. This also provides us with natural validation of cosparse analysis model.

If the sources are not moving, the set of zeroes is very structured: at most spatial locations, $f(\mathbf{r}, t)=0$ for all times $t$. This motivates the introduction of structured cosparsity that will be developed further below for source localization.

Often, the quantity $u$ also satisfies some initial conditions and some boundary conditions. In fact, $u$ is completely determined by the initial conditions, the boundary conditions and the equation $\mathbf{L} u=f$. A special but common case is when the conditions take a homogeneous form, e.g., Neumann boundary conditions for the wave equation. After proper discretization this can be written generically as

$$
\mathbf{C}_{\mathbf{I}} \mathbf{x}=0, \mathbf{C}_{\mathbf{B}} \mathbf{x}=0
$$

for some linear operators $\mathbf{C}_{\mathbf{I}}, \mathbf{C}_{\mathbf{B}}$.

Observations of the physical quantity $u$ by a number of sensors are often modeled as a linear process where the measures, $\mathbf{y}$, are related to $\mathbf{x}$ as $\mathbf{y} \approx \mathbf{M x}$. Given the underlying physics, $\Omega \mathrm{x}$ has many zeroes, hence it is cosparse. Since $\mathbf{C}_{\mathbf{I}} \mathbf{x}=0, \mathbf{C}_{\mathbf{B}} \mathbf{x}=0$, a cosparse approach to estimate $\mathbf{x}$ consists in seeking $\mathrm{x}$ such that $\Omega \mathrm{x}$ has many zeroes, with the constraint

$$
\left[\begin{array}{l}
\mathbf{y} \\
0 \\
0
\end{array}\right] \approx\left[\begin{array}{l}
\mathrm{M} \\
\mathrm{C}_{\mathrm{I}} \\
\mathrm{C}_{\mathrm{B}}
\end{array}\right] \mathrm{x} .
$$

\subsection{An Example: Discretization of Wave Equation}

For simplicity, we consider the pressure field $u$ defined on a 2-dimensional plate $D$ over time. Let $\mathbf{x}$ be the samples of the field $u$ on some uniformly spaced grid points of $D$ at regular time intervals. Then, Eq. (1) leads us to conclude that

$$
\omega_{i j k} \mathbf{x}:=\left(\left(d_{1}+d_{2}\right) \mathbf{x}\right)_{i j k}-\frac{1}{\tilde{c}^{2}}\left(d_{3} \mathbf{x}\right)_{i j k} \approx 0
$$

whenever $(i, j)$ corresponds to a location away from the sources or $k$ is associated to the time when the sources are not active. Here, $d_{l}$ denotes the second-order difference in the $l$-th index, e.g., $\left(d_{2} \mathbf{x}\right)_{i j k}:=\mathbf{x}_{i, j+1, k}-2 \mathbf{x}_{i j k}+\mathbf{x}_{i, j-1, k}$, and the constant $\tilde{c}$ depends on the constant $c$ and the sampling conditions. Hence, with $\boldsymbol{\Omega}$ defined as the collection of all row vectors $\omega_{i j k}$, the signal $\mathbf{x}$ satisfies the cosparse model.

\section{THE GRASP ALGORITHM: STRUCTURED COSPARSITY}

In this section, we describe an extension of the GAP algorithm $[2,3]$ that exploits structured cosparsity to solve inverse problems.

The aim of the GAP algorithm is to find the signal $\mathbf{x} \in \mathbf{R}^{d}$ that is maximally cosparse with respect to $\Omega$ given a linear observation $\mathbf{y} \approx \mathbf{M x}$ for some known $\mathbf{M} \in \mathbf{R}^{m \times d}$. This is achieved by identifying the rows $\Lambda$ of $\Omega$ such that $\Omega_{\Lambda} \mathrm{x}=0$.

In the setting of Section 2.2, due to the assumption that the sources are not moving and there are few of them, we note that for most locations $(i, j)$, the analysis coefficient $\omega_{i j k} \mathbf{x}$ is (close to) zero for all times $k$. Hence, for such $(i, j)$ 's,

$$
\sum_{k}\left|\omega_{i j k} \mathbf{x}\right|^{2}=0
$$

that is, groups of analysis coefficients are simultaneously zero. We call such relations among analysis coefficients the 'structured cosparsity.' This is reminiscent of similar notions in the synthesis framework $[11,12,13]$. The structured cosparsity in our setting is quite natural because it is tied to the physical phenomenon that the sources are not moving.

We exploit structured cosparsity in the GAP straightforwardly. We form groups $\left\{G_{g}\right\}_{g \in I}$ of indices $G_{g} \subset \llbracket 1, p \rrbracket:=$ $\{1, \ldots, p\}$. For example, if the first and second rows of $\boldsymbol{\Omega}$ tend to lead to simultaneous zero entries of representations, then one could take $G_{1}=\{1,2\}$. We require neither $G_{g}$ 's to be disjoint nor to cover the whole $\llbracket 1, p \rrbracket$. Algorithm 1 describes the structured version of GAP, the GRASP. We remark that when $G_{g}$ 's are taken to be all the singletons of $\llbracket 1, p \rrbracket$, the GRASP reduces to the original GAP algorithm.

\section{EXPERIMENT}

We now apply GAP/GRASP to a toy problem of source localization and field reconstruction as discussed in Section 2.2. 


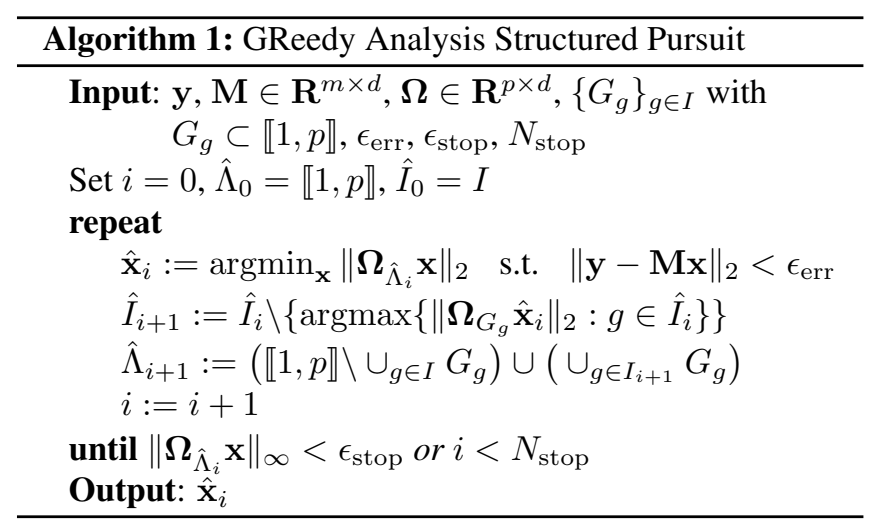

\subsection{Construction of cosparse signals}

Due to computational difficulties, we selected a rather small size for our experiment: A rectangular grid of size $N=$ $11 \times 21=231$ was chosen to represent our plate $D$, and the number of time samples was set to $K=40$ or $K=120$. Furthermore, we enforce the approximate equality in Eq. (5) to be exact equality, i.e., $\omega_{i j k} \mathbf{x}=0$.

To construct cosparse pressure fields, the source locations $S$ were randomly chosen to be interior grid points of $D$. Let $\mathbf{z}$ be such that $\mathbf{z}_{i j k}$ 's are independent random Gaussian for $(i, j) \in S$ and $k=2, \ldots, K_{s}+1$ for some $K_{s}<K$, and is zero everywhere else. For our experiment, we chose $K_{s}=$ 20 . We simulate the sources by imposing $\Omega \mathbf{x}=\mathbf{z}$. Therefore, $\omega_{i j k} \mathbf{x}=0$ for all $k>K_{s}+1$. This was exploited in the GRASP; $(i, j, k)$ for $k>K_{s}+1$ were not included in $G_{g}$ 's in Algorithm 1. We used the Neumann boundary conditions: $\mathbf{x}_{1 j k}=\mathbf{x}_{2 j k}, \mathbf{x}_{11, j, k}=\mathbf{x}_{10, j, k}$, etc., were enforced through the form $\mathbf{C}_{\mathbf{B}} \mathbf{x}=0$. Initial conditions were $\mathbf{x}_{i j 1}=\mathbf{x}_{i j 2}=0$ for all $(i, j)$, enforced through the form $\mathbf{C}_{\mathbf{I}} \mathbf{x}=0$.

To build a cosparse signal $\mathrm{x}$, we need to solve $\Omega \mathrm{x}=\mathrm{z}$, $\mathbf{C}_{\mathbf{I}} \mathbf{x}=0$, and $\mathbf{C}_{\mathbf{B}} \mathbf{x}=0$. Fortunately, $\boldsymbol{\Omega} \mathbf{x}=\mathbf{z}$ is a causal relation in the sense that if $\mathbf{x}$ is known for $k=k_{0}-1, k_{0}-2$, then $\mathrm{x}$ for $k=k_{0}$ can be uniquely and efficiently computed. Indeed, this was how we constructed the cosparse vector $\mathbf{x}$ rather than employing general matrix inversion techniques.

\subsection{Evaluation of GRASP}

A typical source localization problem is as follows: Given a limited number of measurements of $\mathbf{x}$ obtained from a few microphones, $\mathbf{y}=\mathbf{M x}$, how to locate the source locations? Furthermore, can we reconstruct $\mathbf{x}$ given the "extended" constraint (4) ? For our simulation, we chose random locations in $D$ that do not coincide with points in $S$, and assumed that the values of $\mathbf{x}$ were measured at those locations at all times.

We varied the number of sources $s$ and the number of measurements $m$, and repeated source localization and field reconstruction with GRASP 30 times for each pair $(s, m)$.

As performance measures, we observed what portion of the source locations were correctly identified and how often
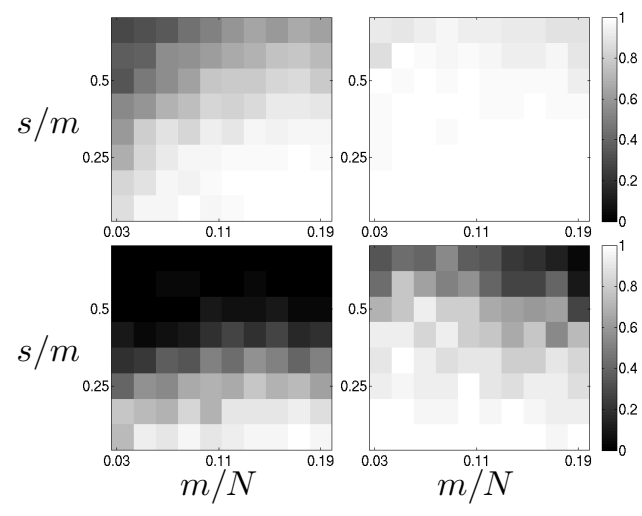

Fig. 1. Average portion of source locations identified (top) and average recovery rate of the pressure field $\mathrm{x}$ (bottom). Time samples were $K=40$ (left) and $K=120$ (right).

the signal $\mathbf{x}$ was recovered. We regarded $\mathbf{x}$ as perfectly recovered when all the sources were located (no groups $G_{g}$ left at the end of Algorithm 1 corresponded to points of $S$ ) and the relative $\ell_{2}$-norm error of $\hat{\mathbf{x}}$ from the GRASP is within 0.1 .

Fig. 1 shows average portion of source locations correctly identified and average recovery rate of $\mathrm{x}$ by the GRASP. We notice that measurements taken over longer period helps the performance. When the measurements were collected for long duration $K=120$, the source localization results were quite encouraging; over $93 \%$ of source locations were correctly identified in all cases. While less impressive, we observe that the recovery rate is still promising when there are few sources active (bottom row in the diagrams). It is important to note that we are not concerned with false alarms; when the GRASP picks a location to remove, it does not mean that the location is classified to be a source. That decision is made only from the final estimate $\hat{x}$ from the GRASP and its analysis representation.

\subsection{Structured cosparsity: what is the gain?}

We also applied GRASP and GAP to a cosparse pressure field reconstruction problem where one source was active and 12 microphones were used, to gauge the benefit of using structured cosparsity. Out of 20 experiments, the GRASP successfully recovered all the sources 18 times, and in those cases, the means of the relative errors on $\mathrm{x}$ were 0.014 for the GRASP versus 0.112 for the GAP. Considering that the GAP also failed when the GRASP did (bad relative errors for both algorithms), we conclude - though from a limited experimental result due to computational issue (more from the GAP) - that there is clear advantage in the use of structured cosparsity.

\section{DISCUSSION AND CONCLUSION}

The primary goal of this paper is to show how cosparsity "naturally" arises via discretization when well-understood physi- 
cal laws govern the data of interest. By this process, appropriate cosparsifying operators can be designed and exploited.

The potential of such physics-driven cosparse models was illustrated in the context of source localization with acoustic waves, a scenario that has been widely studied. A fair assessment of the merits of cosparsity in this scenario requires extensive comparisons that will be the object of further work.

Sparsity vs cosparsity. In the considered scenario, the cosparse model exploits linear equations that uniquely characterize the unknown pressure field $\mathbf{x}$. Hence, the inversion of the associated linear system yields a sparse model $\mathbf{x}=\mathbf{D} s$ where $s$ is the sparse source term. Therefore, in this context, cosparsity and sparsity are equivalent models. Indeed, standard sparse regularization $[14,15,16]$ can be used to locate sources or to reconstruct $\mathbf{x}$ from $\mathbf{y}=\mathbf{M D} s$. However, there seems to be computational differences as well as extended scenarios where the cosparse model is no longer equivalent to the sparse one and offers new perspectives.

Extended scenario: unknown boundaries? Suppose we observe $\mathbf{y}=\mathbf{M x}$ where the pressure field $\mathbf{x}$ satisfies the wave equation, with few sources, in a domain whose boundaries are a priori unknown. For example, it may have been recorded either in a small room with boundary conditions $\mathbf{C}_{\mathbf{B}_{1}} \mathbf{x}=0$ or a larger one with boundary conditions $\mathbf{C}_{\mathbf{B}_{\mathbf{2}}} \mathbf{x}=$ 0 . In this context it is hard to express a natural sparse model with a dictionary of Green functions, but one can imagine a natural structured cosparse model where the analysis operator is obtained by concatenating $\boldsymbol{\Omega}$ and $\mathbf{C}_{\mathbf{B}_{i}}$ for all possible room configurations (one may imagine also a "continuum" of room sizes and shapes, e.g., convex shaped rooms). In upcoming work we will explore the potential of this approach to simultaneously locate sources and determine the geometry of the domain (shape/location/nature of the boundary conditions).

Computational pitfalls, challenges, and opportunities. The matrix MD arising in the standard sparse model is essentially a collection of Green functions between $m$ sensor locations and all possible source locations. Its pre-computation amounts to solving the wave equation $m$ times, and storing the resulting spatio-temporal fields. The required resources can become exceedingly costly, e.g., for large spatial domains with complex geometries and reverberant boundary conditions. Moreover, sparse reconstruction algorithms iteratively apply the operators $\mathbf{M D}$ and $\mathbf{D}^{T} \mathbf{M}^{T}$ with a potentially high computational cost. Computational challenges also arise with the cosparse approach, but they seem to be of a different nature: unlike $\mathbf{D}$, the operators $\Omega, \mathbf{C}_{\mathbf{I}}, \mathbf{C}_{\mathbf{B}}$ are very sparse, and can be implicitly implemented without being stored in matrix form. The major computational challenge in applying GAP/GRASP is in iteratively solving least squares problems involving these operators. In this paper, these were solved in the time-domain with generic numerical linear algebra. We expect that working in the Fourier domain and exploiting the sparse spatial structure of $\Omega$ will provide substantial speedups, allowing to test the approach at a much larger scale with real data and more accurate discretization of the underlying physical model.

\section{REFERENCES}

[1] M. Elad, P. Milanfar, and R. Rubinstein, "Analysis versus synthesis in signal priors," Inverse Problems, vol. 23, no. 3, pp. 947-968, June 2007.

[2] S. Nam, M. E. Davies, M. Elad, and R. Gribonval, "The Cosparse Analysis Model and Algorithms," Research report, 2011, Submitted.

[3] S. Nam, M. E. Davies, M. Elad, and R. Gribonval, "Recovery of cosparse signals with greedy analysis pursuit in the presence of noise," in CAMSAP Proc., Dec. 2011.

[4] J. Portilla, "Image restoration through 10 analysis-based sparse optimization in tight frames," Proc. of ICIP, pp. 3865-3868, 2009.

[5] I. W. Selesnick and M. A. T. Figueiredo, "Signal restoration with overcomplete wavelet transforms: Comparison of analysis and synthesis priors," SPIE Proc., vol. 7446 (Wavelets XIII), August 2009.

[6] J.-L. Starck, E.J. Candès, and D.L. Donoho, "The curvelet transform for image denoising," IEEE Trans. Image Processing, vol. 11, no. 11, pp. 670 684, Nov. 2002.

[7] D. Labate, W.-Q Lim, G. Kutyniok, and G. Weiss, "Sparse multidimensional representation using shearlets," in Wavelets XI (San Diego, CA, 2005), 254-262, SPIE Proc. 5914, Bellingham, WA, 2005, pp. 254-262.

[8] B. Ophir, M. Elad, N. Bertin, and M. D. Plumbley, "Sequential Minimal Eigenvalues - An Approach to Analysis Dictionary Learning," in EUSIPCO Proc., Barcelona, Spain, 2011.

[9] M. Yaghoobi, S. Nam, R. Gribonval, and M. E. Davies, "Analysis Operator Learning for Overcomplete Cosparse Representations," in EUSIPCO Proc., Barcelona, Spain, 2011.

[10] G. Peyré and J. Fadili, "Learning analysis sparsity priors," in Proc. of Sampta'11, 2011.

[11] R. Jenatton, J.-Y. Audibert, and F. Bach, "Structured Variable Selection with Sparsity-Inducing Norms," Journal of Machine Learning Research, vol. 12, pp. 2777-2824, 2011.

[12] Y. C. Eldar and M. Mishali, "Robust recovery of signals from a structured union of subspaces," IEEE Trans. Info. Theory, vol. 55, no. 11, pp. 5302-5316, 2009.

[13] R. G. Baraniuk, V. Cevher, M. F. Duarte, and C. Hegde, "Model-based compressive sensing," IEEE Trans. Info. Theory, vol. 56, no. 4, pp. 1982-2001, 2010.

[14] D. Malioutov, M. Cetin, and A.S. Willsky, "A sparse signal reconstruction perspective for source localization with sensor arrays," IEEE Trans. Signal Processing, vol. 53, no. 8, pp. 3010 - 3022, aug. 2005.

[15] D. Model and M. Zibulevsky, "Signal reconstruction in sensor arrays using temporal-spatial sparsity regularization," in Independent Component Analysis and Blind Signal Separation, Carlos Puntonet and Alberto Prieto, Eds., vol. 3195 of Lecture Notes in Computer Science, pp. 319-326. Springer Berlin / Heidelberg, 2004.

[16] V. Cevher, M. Duarte, and R. Baraniuk, "Distributed localization via spatial sparsity," in EUSIPCO Proc., 2008. 